Article

\title{
Various Extraction Methods for Obtaining Stilbenes from Grape Cane of Vitis vinifera $\mathrm{L}$.
}

\author{
Ivo Soural ${ }^{1, *}$, Naděžda Vrchotová ${ }^{2}$, Jan Tříska ${ }^{2}$, Josef Balík ${ }^{1}$, Štěpán Horník ${ }^{3}$, \\ Petra Cuř́nová ${ }^{3}$ and Jan Sýkora ${ }^{3}$
}

1 Department of Post-Harvest Technology of Horticultural Products, Faculty of Horticulture, Mendel University in Brno, Valtická 337, Lednice 69144, Czech Republic;

E-Mail: josef.balik@mendelu.cz

2 Global Change Research Centre, Academy of Sciences of the Czech Republic, v. v. i., Branišovská 31, České Budějovice 37005, Czech Republic;

E-Mails: vrchotova.n@czechglobe.cz (N.V.); triska.j@czechglobe.cz (J.T.)

3 Institute of Chemical Process Fundamentals, Academy of Sciences of the Czech Republic, v.v.i.,

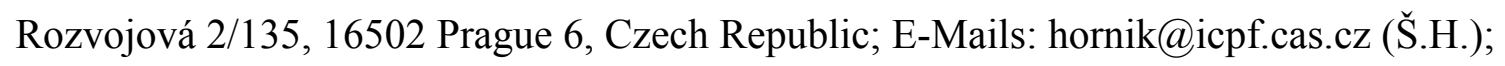
curinova@icpf.cas.cz (P.C.); sykora@icpf.cas.cz (J.S.)

* Author to whom correspondence should be addressed; E-Mail: ivo.soural@mendelu.cz; Tel.: +420-519-367-266; Fax: +420-519-367-222.

Academic Editor: Derek J. McPhee

Received: 16 February 2015 / Accepted: 2 April 2015 / Published: 8 April 2015

\begin{abstract}
Grape cane, leaves and grape marc are waste products from viticulture, which can be used to obtain secondary stilbene derivatives with high antioxidant value. The presented work compares several extraction methods: maceration at laboratory temperature, extraction at elevated temperature, fluidized-bed extraction, Soxhlet extraction, microwave-assisted extraction, and accelerated solvent extraction. To obtain trans-resveratrol, trans- $\varepsilon$-viniferin and $\mathrm{r} 2$-viniferin from grape cane of the $V$. vinifera variety Cabernet Moravia, various conditions were studied: different solvents, using powdered versus cut cane material, different extraction times, and one-step or multiple extractions. The largest concentrations found were $6030 \pm 680 \mu \mathrm{g} / \mathrm{g}$ dry weight (d.w.) for trans-resveratrol, $2260 \pm 90 \mu \mathrm{g} / \mathrm{g} \mathrm{d}$.w. for trans- $\varepsilon$-viniferin, and $510 \pm 40 \mu \mathrm{g} / \mathrm{g} \mathrm{d}$.w. for r2-viniferin. The highest amounts of stilbenes $(8500 \pm 1100 \mu \mathrm{g} / \mathrm{g}$ d.w. $)$ were obtained using accelerated solvent extraction in methanol.
\end{abstract}


Keywords: Vitis vinifera L.; grape cane; stilbenes; accelerated solvent extraction (ASE); microwave-assisted extraction (MAE); LC-MS

\section{Introduction}

Resveratrol has been studied intensively in recent decades as one of the major stilbene derivatives [1,2]. It is believed that this substance bears responsibility for the French paradox connected with wine consumption [3,4]. In addition to resveratrol, the stilbene derivative family encompasses such other biologically active compounds as viniferins [5], piceid [6], pinosylvin [7] and pterostilbene [8], to mention just the most important ones [9]. Wine production and viticulture technology produce four types of waste plant material: grape canes, leaves [10], grape marc and young lateral shoots. According to the literature, stilbenes contained in waste material from wine production are good sources of these substances for the pharmaceutical, cosmetic, and food industries [11]. In order to maximize yields of these compounds from viticulture and wine production byproducts, development of improved methods is required.

It is known from the literature that some of the best solvents for stilbene extraction are alcohols (methanol or ethanol) from the protic group [11]. We studied extraction mainly using methanol as a protic solvent because of its physical properties, and we compared its extraction power with acetone as an aprotic solvent. In some cases we performed additional extraction steps to improve yields, because it has been observed, for example, that from the first extraction of stilbenes from grape cane only approximately $40 \%$ of total stilbenes were obtained, while the total yield of stilbenes from the first through fourth extractions was around 95\% [12].

Our work focused on studying and comparing various methods of extracting stilbenes from grape canes, including maceration at laboratory temperature, higher temperature extraction, fluidized-bed extraction, Soxhlet extraction, microwave-assisted extraction, and accelerated solvent extraction.

\section{Results and Discussion}

In our experiments, we focused on different extraction methods using various conditions: solvents, sizes of material, temperatures, times, and multiple extractions. In most extractions two solvents were compared - methanol and acetone-for extracting mainly stilbenes: trans-resveratrol, trans- $\varepsilon$-viniferin (a dimer of trans-resveratrol), and r2-viniferin (a tetramer of trans-resveratrol). All three compounds were determined by liquid chromatography using diode array detector/fluorescence detector (DAD/FLD) and low-resolution liquid chromatography-mass spectrometry with ion trap (LCMS-IT) system as described in the Experimental section below. Next to LC-DAD/FLD and low resolution LCMS-IT, both viniferins were identified by high-resolution mass spectrometry (HRMS) and by nuclear magnetic resonance (LC-NMR). The results for all studied parameters are detailed in the following paragraphs. 


\subsection{Absolute Concentration}

The highest values were obtained from grape cane of Vitis vinifera $\mathrm{cv}$. Cabernet Moravia were $6030 \pm 680 \mu \mathrm{g} / \mathrm{g}$ dry weight (d.w.) of trans-resveratol, $2260 \pm 90 \mu \mathrm{g} / \mathrm{g} \mathrm{d}$.w. of trans- $\varepsilon$-viniferin, and $510 \pm 40 \mu \mathrm{g} / \mathrm{g}$ d.w. of $\mathrm{r} 2$-viniferin. Overview of the extraction methods is presented in Table 1 and the amount of each above-mentioned stilbene obtained by extraction procedures 1-25 is given in Figure 1 . Comparing contents of trans-resveratrol and trans- $\varepsilon$-viniferin with the literature [11] (3.45 and $1.30 \mathrm{mg} / \mathrm{g}$ d.w., cv. Pinot Noir) and [5] (4.7 and $1.7 \mathrm{mg} / \mathrm{g} \mathrm{dw}$, cv. Hasaine Sladki), shows a similar ratio of around 2.7 between trans-resveratrol and trans- $\varepsilon$-viniferin. The highest total concentration of all three stilbenes $(8500 \pm 1100 \mu \mathrm{g} / \mathrm{g}$ d.w. $)$ was obtained using accelerated solvent extraction in methanol. The higher amount of trans-resveratrol in comparison with the two viniferins was from extracting at laboratory temperature from cut grape cane. In that case, the concentration of trans-resveratrol was 6.85 times higher than the sum of trans- $\varepsilon$-viniferin and $\mathrm{r} 2$-viniferin.

Table 1. Overview of extraction methods (number, abbreviation and description).

\begin{tabular}{|c|c|c|}
\hline No. & $\begin{array}{l}\text { Short Terms } \\
\text { (Legends) }\end{array}$ & $\begin{array}{l}\text { Descriptions of Extraction Methods } \\
\text { [Type and Temp./Time or Step Extraction/Material/Solvent] }\end{array}$ \\
\hline$\{1\}$ & Lab. T. Acet. 8 h (C) & Laboratory temperature, $8 \mathrm{~h}$, cut, acetone \\
\hline$\{2\}$ & Lab. T. Acet. 2 d (C) & Laboratory temperature, 2 days, cut, acetone \\
\hline$\{3\}$ & Lab. T. Acet. 4 d (C) & Laboratory temperature, 4 days, cut, acetone \\
\hline$\{4\}$ & Lab. T. Acet. 7 d (C) & Laboratory temperature, 7 days, cut, acetone \\
\hline$\{5\}$ & Lab. T. MeOH 8 h (C) & Laboratory temperature, $8 \mathrm{~h}$, cut, methanol \\
\hline$\{6\}$ & Lab. T. MeOH 2 d (C) & Laboratory temperature, 2 days, cut, methanol \\
\hline$\{7\}$ & Lab. T. MeOH 4 d (C) & Laboratory temperature, 4 days, cut, methanol \\
\hline$\{8\}$ & Lab. T. MeOH 7 d (C) & Laboratory temperature, 7 days, cut, methanol \\
\hline$\{9\}$ & Lab. T. MeOH 7 d (P) & Laboratory temperature, 7 days, powdered, methanol \\
\hline$\{10\}$ & $50{ }^{\circ} \mathrm{C}$ Acet. $(\mathrm{P})$ & Increased temperature $50^{\circ} \mathrm{C}, 2.75 \mathrm{~h}$, powdered, acetone \\
\hline$\{11\}$ & $50^{\circ} \mathrm{C} \mathrm{MeOH}(\mathrm{P})$ & Increased temperature $50^{\circ} \mathrm{C}, 2.75 \mathrm{~h}$, powdered, methanol \\
\hline$\{12\}$ & FBE Acet. (P) & FBE (boiling temperature), $100 \mathrm{~min}$, powdered, acetone \\
\hline$\{13\}$ & FBE $\mathrm{MeOH}(\mathrm{P})$ & FBE (boiling temperature), $100 \mathrm{~min}$, powdered, methanol \\
\hline$\{14\}$ & $\mathrm{FBE} \mathrm{MeOH}(\mathrm{C})$ & FBE (boiling temperature), $100 \mathrm{~min}$, cut, methanol \\
\hline$\{15\}$ & Reflux Acet. $1 \mathrm{~h}(\mathrm{P})$ & Reflux (boiling temperature), $1 \mathrm{~h}$, powdered, acetone \\
\hline$\{16\}$ & Reflux MeOH 1 h (P) & Reflux (boiling temperature), $1 \mathrm{~h}$, powdered, methanol \\
\hline$\{17\}$ & Reflux $\mathrm{MeOH} 1 \mathrm{~h}(\mathrm{C})$ & Reflux (boiling temperature), $1 \mathrm{~h}$, cut, methanol \\
\hline$\{18\}$ & Reflux MeOH 2 h (P) & Reflux (boiling temperature), $2 \mathrm{~h}$, powdered, methanol \\
\hline$\{19\}$ & Soxhlet MeOH 1st (P) & Soxhlet (boiling temperature), 1 st step, powdered, methanol \\
\hline$\{20\}$ & Soxhlet $\mathrm{MeOH}$ 2nd (P) & Soxhlet (boiling temperature), 2nd step, powdered, methanol \\
\hline$\{21\}$ & Soxhlet $\mathrm{MeOH}$ 3rd $(\mathrm{P})$ & Soxhlet (boiling temperature), 3rd step, powdered, methanol \\
\hline$\{22\}$ & Soxhlet $\mathrm{MeOH} 4$ th $(\mathrm{P})$ & Soxhlet (boiling temperature), 4th step, powdered, methanol \\
\hline$\{23\}$ & Soxhlet $\mathrm{MeOH} 5$ th $(\mathrm{P})$ & Soxhlet (boiling temperature), 5th step, powdered, methanol \\
\hline$\{24\}$ & MAE MeOH (P) & MAE (boiling temperature), $30 \mathrm{~min}$, powdered, methanol \\
\hline$\{25\}$ & $\mathrm{ASE} \mathrm{MeOH}(\mathrm{P})$ & ASE $\left(100^{\circ} \mathrm{C}\right), 15 \mathrm{~min}$, powdered, methanol \\
\hline
\end{tabular}




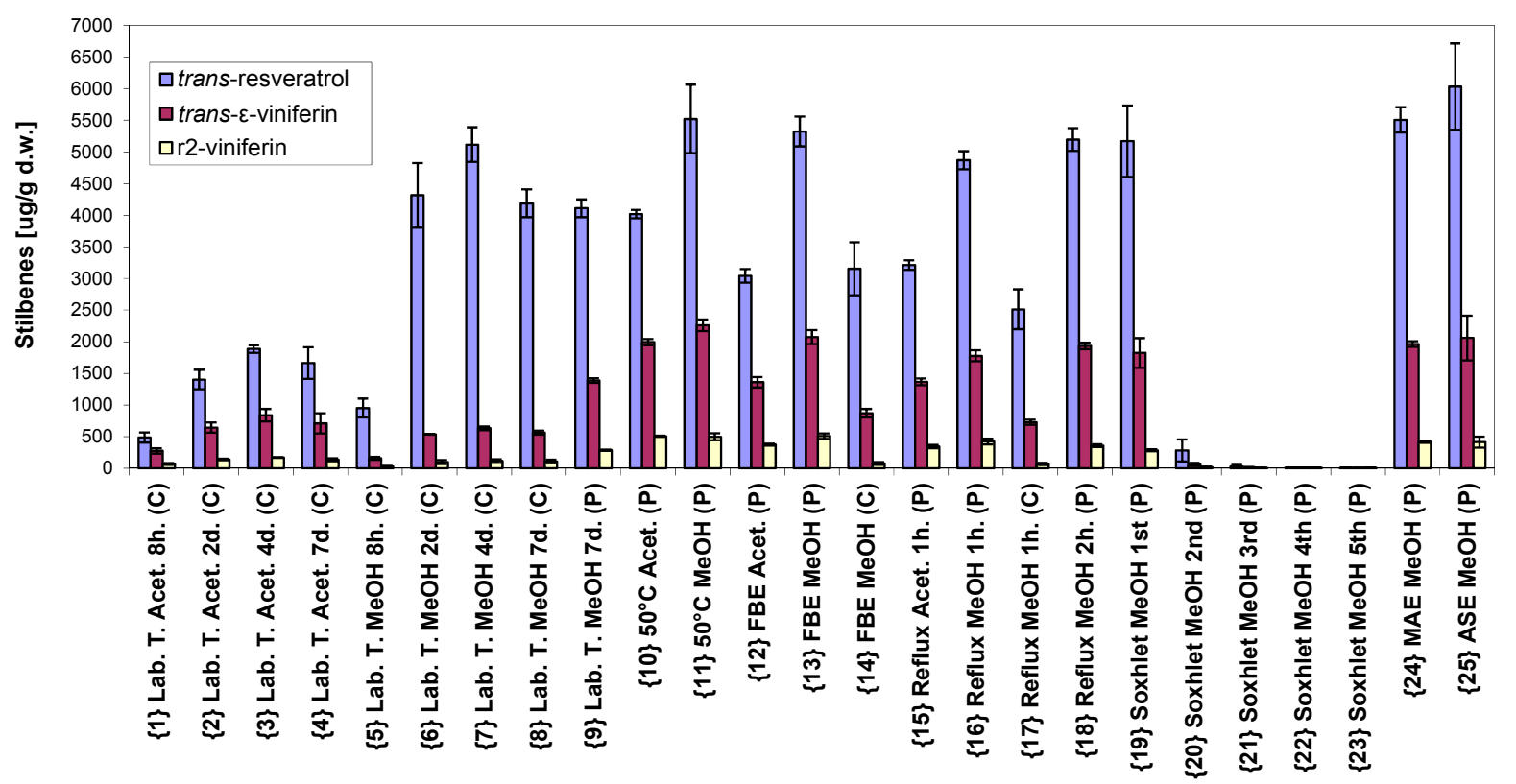

Figure 1. Concentration of trans-resveratrol, trans- $\varepsilon$-viniferin and $\mathrm{r} 2$-viniferin in $\mu \mathrm{g} / \mathrm{g}$ dry weight (d.w.) obtained using different extraction procedures (abbreviations are described in Table 1).

\subsection{The Highest Yields}

The main extraction results are summarized in Figure 2. From the graph, it can be seen that the largest amount of trans-resveratrol was obtained by accelerated solvent extraction (ASE) using methanol as solvent and powdered source material, the highest yield for trans- $\varepsilon$-viniferin was obtained by extraction at $50{ }^{\circ} \mathrm{C}$, and the highest for $\mathrm{r} 2$-viniferin using fluidized-bed extraction with methanol.

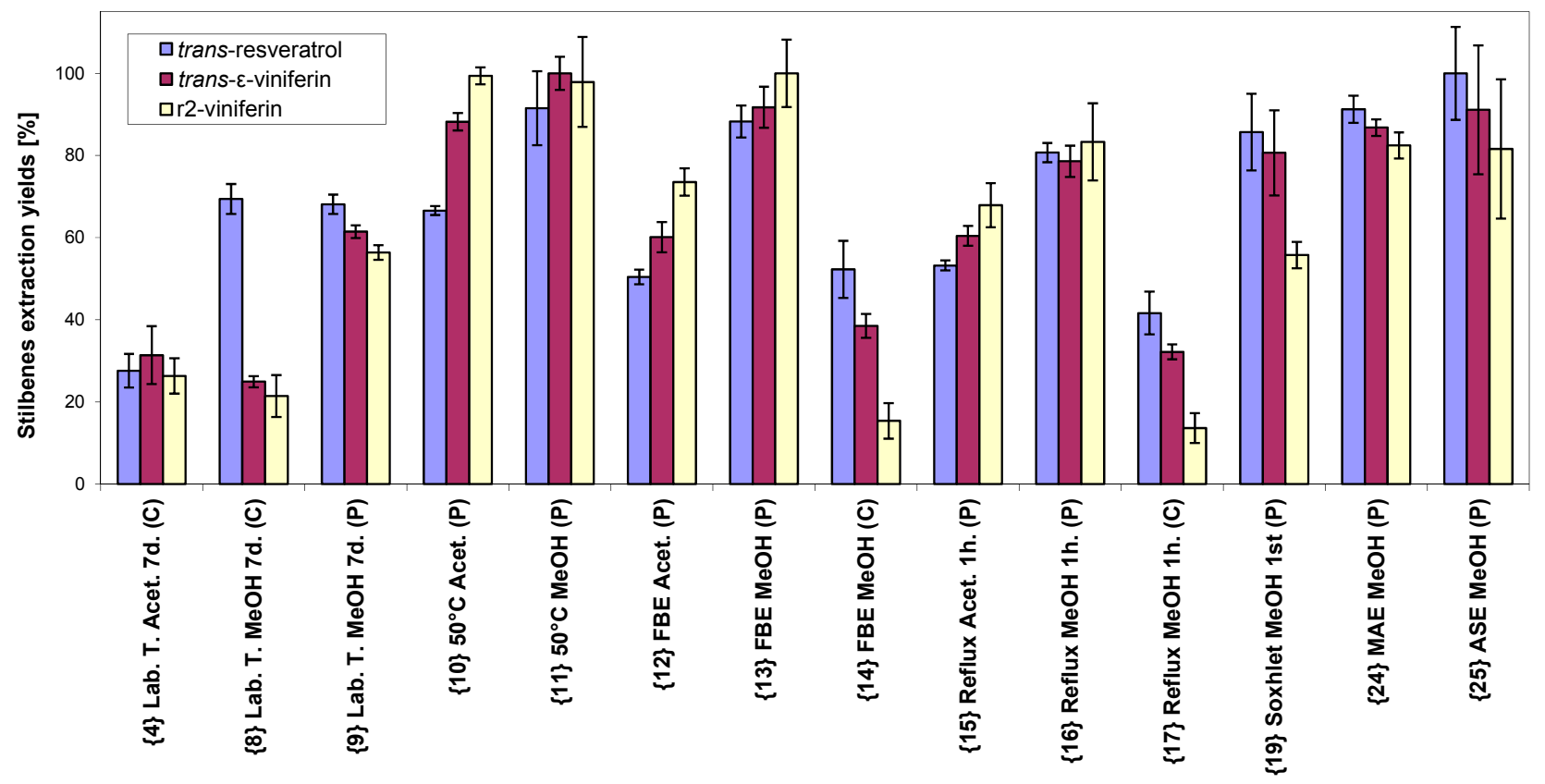

Figure 2. Comparison of extraction yields for stilbenes from different extractions methods (abbreviations are described in Table 1). 
If we set $70 \%$ as a lowest limit for acceptable extraction yield for all three compounds, then the following extraction methods provide almost the same yield: at $50{ }^{\circ} \mathrm{C}$ for methanol and acetone; extraction by ASE; microwave-assisted extraction (MAE); extraction by fluidized-bed extraction with methanol; and extraction with methanol heated to reflux.

\subsection{The Effect of Conditions on Extraction of Stilbenes}

To obtain all three stilbenes from grape cane of the $V$. vinifera variety Cabernet Moravia, various conditions were studied: different solvents, using powdered versus cut material, different temperatures, different extraction times, and one-step or multiple extractions.

\subsubsection{Solvents}

Methanol generally extracted grapevine canes better than acetone in these experiments, and the extraction yield with methanol in percentages of each compound was decreasing in the following order: trans-resveratrol, trans- $\varepsilon$-viniferin and r2-viniferin. The opposite situation was true for acetone, in which case the percentage values of trans-resveratrol were the smallest. The greatest difference between methanol and acetone occurred when extracting of cut grape canes at laboratory temperature; in this case extracts from acetone had very small concentrations of trans-resveratrol in comparison with those from methanol, but the contents of trans-e-viniferin and r2-viniferin were a little smaller in methanol than in acetone. A statistical analysis is presented in detail in Table 2.

Table 2. Tukey's honest significance test for stilbenes (trans-resveratrol, trans- $\varepsilon$-viniferin and $\mathrm{r} 2$-viniferin) in $\mu \mathrm{g} / \mathrm{g} \mathrm{d}$.w. for different solvents. (Two-way ANOVA: $* * p<0.01$, $* p<0.05$, n.s. $=$ not significant. Values are means and standard deviations calculated from three measurements).

\begin{tabular}{|c|c|c|c|c|c|c|c|c|c|c|c|c|c|c|}
\hline Extraction & $\{1\}$ & $\{2\}$ & $\{3\}$ & $\{4\}$ & $\{15\}$ & $\{10\}$ & $\{12\}$ & $\{5\}$ & $\{6\}$ & $\{7\}$ & $\{8\}$ & $\{16\}$ & $\{11\}$ & $\{13\}$ \\
\hline Resveratrol & 486.9 & 1401.9 & 1884.9 & 1663.3 & 3210.8 & 4016.5 & 3041.1 & 951.4 & 4314.1 & 5115.4 & 4189.0 & 4868.5 & 5521.6 & 5324.1 \\
\hline Viniferin & 275.2 & 643.7 & 838.2 & 709.5 & 1366.3 & 1994.4 & 1359.1 & 157.9 & 536.0 & 632.1 & 563.1 & 1776.7 & 2260.3 & 2074.1 \\
\hline r2-Viniferin & 67.0 & 139.8 & 170.1 & 133.5 & 344.7 & 504.5 & 373.3 & 27.2 & 98.3 & 115.1 & 108.7 & 422.7 & 496.9 & $\mathbf{5 0 7 . 5}$ \\
\hline \multicolumn{15}{|l|}{1} \\
\hline \multicolumn{15}{|l|}{ Lab. T. Acet. } \\
\hline \multicolumn{15}{|l|}{$8 \mathrm{~h}(\mathrm{C})$} \\
\hline$\{2\}$ & $* *$ & & & & & & & & & & & & & \\
\hline Lab. T. Acet. & $* *$ & & & & & & & & & & & & & \\
\hline $2 \mathrm{~d}(\mathrm{C})$ & n.s. & & & & & & & & & & & & & \\
\hline$\{3\}$ & $* *$ & n.s. & & & & & & & & & & & & \\
\hline Lab. T. Acet. & $* *$ & n.s. & & & & & & & & & & & & \\
\hline $4 \mathrm{~d}(\mathrm{C})$ & $*$ & n.s. & & & & & & & & & & & & \\
\hline$\{4\}$ & $* *$ & n.s. & n.s. & & & & & & & & & & & \\
\hline Lab. T. Acet. & $* *$ & n.s. & n.s. & & & & & & & & & & & \\
\hline $7 \mathrm{~d}(\mathrm{C})$ & n.s. & n.s. & n.s. & & & & & & & & & & & \\
\hline$\{15\}$ & $* *$ & $* *$ & $* *$ & $* *$ & & & & & & & & & & \\
\hline Reflux Acet. & $* *$ & $* *$ & $* *$ & $* *$ & & & & & & & & & & \\
\hline $1 \mathrm{~h}(\mathrm{P})$ & $* *$ & $* *$ & $* *$ & $* *$ & & & & & & & & & & \\
\hline
\end{tabular}


Table 2. Cont.

\begin{tabular}{|c|c|c|c|c|c|c|c|c|c|c|c|c|c|c|}
\hline Extraction & $\{1\}$ & $\{2\}$ & $\{3\}$ & $\{4\}$ & $\{15\}$ & $\{10\}$ & $\{12\}$ & $\{5\}$ & $\{6\}$ & $\{7\}$ & $\{8\}$ & $\{16\}$ & $\{11\}$ & $\{13\}$ \\
\hline Resveratrol & 486.9 & 1401.9 & 1884.9 & 1663.3 & 3210.8 & 4016.5 & 3041.1 & 951.4 & 4314.1 & 5115.4 & 4189.0 & 4868.5 & 5521.6 & 5324.1 \\
\hline Viniferin & 275.2 & 643.7 & 838.2 & 709.5 & 1366.3 & 1994.4 & 1359.1 & 157.9 & 536.0 & 632.1 & 563.1 & 1776.7 & 2260.3 & 2074.1 \\
\hline r2-Viniferin & 67.0 & 139.8 & 170.1 & 133.5 & 344.7 & 504.5 & 373.3 & 27.2 & 98.3 & 115.1 & 108.7 & 422.7 & 496.9 & 507.5 \\
\hline$\{10\}$ & $* *$ & $* *$ & $* *$ & $* *$ & $*$ & & & & & & & & & \\
\hline $50^{\circ} \mathrm{C}$ Acet. & $* *$ & $* *$ & $* *$ & $* *$ & $* *$ & & & & & & & & & \\
\hline (P) & $* *$ & $* *$ & $* *$ & $* *$ & $* *$ & & & & & & & & & \\
\hline$\{12\}$ & $* *$ & $* *$ & $* *$ & $* *$ & n.s. & $* *$ & & & & & & & & \\
\hline FBE Acet. & $* *$ & $* *$ & $* *$ & $* *$ & n.s. & $* *$ & & & & & & & & \\
\hline$(\mathrm{P})$ & $* *$ & $* *$ & $* *$ & $* *$ & n.s. & $* *$ & & & & & & & & \\
\hline$\{5\}$ & n.s. & n.s. & $* *$ & n.s. & $* *$ & $* *$ & $* *$ & & & & & & & \\
\hline Lab. T. & n.s. & $* *$ & $* *$ & $* *$ & $* *$ & $* *$ & $* *$ & & & & & & & \\
\hline $\mathrm{MeOH} 8 \mathrm{~h}(\mathrm{C})$ & n.s. & $* *$ & $* *$ & $* *$ & $* *$ & $* *$ & $* *$ & & & & & & & \\
\hline$\{6\}$ & $* *$ & $* *$ & $* *$ & $* *$ & $* *$ & n.s. & $* *$ & $* *$ & & & & & & \\
\hline Lab. T. & $*$ & n.s. & $* *$ & n.s. & $* *$ & $* *$ & $* *$ & $* *$ & & & & & & \\
\hline $\mathrm{MeOH} 2 \mathrm{~d}(\mathrm{C})$ & n.s. & n.s. & n.s. & n.s. & $* *$ & $* *$ & $* *$ & n.s. & & & & & & \\
\hline$\{7\}$ & $* *$ & $* *$ & $* *$ & $* *$ & $* *$ & $* *$ & $* *$ & $* *$ & $*$ & & & & & \\
\hline Lab. T. & $* *$ & n.s. & n.s. & n.s. & $* *$ & $* *$ & $* *$ & $* *$ & n.s. & & & & & \\
\hline $\mathrm{MeOH} 4 \mathrm{~d}(\mathrm{C})$ & n.s. & n.s. & n.s. & n.s. & $* *$ & $* *$ & $* *$ & $*$ & n.s. & & & & & \\
\hline$\{8\}$ & $* *$ & $* *$ & $* *$ & $* *$ & $* *$ & n.s. & $* *$ & $* *$ & n.s. & $* *$ & & & & \\
\hline Lab. T. & $* *$ & n.s. & $*$ & n.s. & $* *$ & $* *$ & $* *$ & $* *$ & n.s. & n.s. & & & & \\
\hline $\mathrm{MeOH} 7 \mathrm{~d}(\mathrm{C})$ & n.s. & n.s. & n.s. & n.s. & $* *$ & $* *$ & $* *$ & n.s. & n.s. & n.s. & & & & \\
\hline$\{16\}$ & $* *$ & $* *$ & $* *$ & $* *$ & $* *$ & $*$ & $* *$ & $* *$ & n.s. & n.s. & n.s. & & & \\
\hline Reflux MeOH & $* *$ & $* *$ & $* *$ & $* *$ & $* *$ & n.s. & $* *$ & $* *$ & $* *$ & $* *$ & $* *$ & & & \\
\hline $1 \mathrm{~h}(\mathrm{P})$ & $* *$ & $* *$ & $* *$ & $* *$ & n.s. & n.s. & n.s. & $* *$ & $* *$ & $* *$ & $* *$ & & & \\
\hline$\{11\}$ & $* *$ & $* *$ & $* *$ & $* *$ & $* *$ & $* *$ & $* *$ & $* *$ & $* *$ & n.s. & $* *$ & n.s. & & \\
\hline $50^{\circ} \mathrm{C} \mathrm{MeOH}$ & $* *$ & $* *$ & $* *$ & $* *$ & $* *$ & $*$ & $* *$ & $* *$ & $* *$ & $* *$ & $* *$ & $* *$ & & \\
\hline$(\mathrm{P})$ & $* *$ & $* *$ & $* *$ & $* *$ & $* *$ & n.s. & $* *$ & $* *$ & $* *$ & $* *$ & $* *$ & n.s. & & \\
\hline$\{13\}$ & $* *$ & $* *$ & $* *$ & $* *$ & $* *$ & $* *$ & $* *$ & $* *$ & $* *$ & n.s. & $* *$ & n.s. & n.s. & \\
\hline FBE MeOH & $* *$ & $* *$ & $* *$ & $* *$ & $* *$ & n.s. & $* *$ & $* *$ & $* *$ & $* *$ & $* *$ & $* *$ & n.s. & \\
\hline$(\mathrm{P})$ & $* *$ & $* *$ & $* *$ & $* *$ & $* *$ & n.s. & $* *$ & $* *$ & $* *$ & $* *$ & $* *$ & n.s. & n.s. & \\
\hline
\end{tabular}

\subsubsection{Powdered Versus Cut Materials}

We compared extraction of cut versus powdered grape cane at laboratory temperature, by fluidized-bed extraction, and extraction at boiling temperature. Higher yields were always obtained with the extraction of powdered material. In the case of cut cane extracted with methanol, there were large differences in the extraction yields for trans-resveratrol, trans- $\varepsilon$-viniferin and $\mathrm{r} 2$-viniferin.

The amount of trans-resveratrol obtained by fluidized-bed extraction of cut material (method 14) was about half of that obtained from powdered material (method 13), but the yield of trans-e-viniferin in method 14 was less than half of that from the powdered material (method 13). The yield of r2-viniferin by fluidized-bed extraction from powdered grape cane (method 13) reached the maximal amount actually contained in the cane (see Figure 2), while the yield of r2-viniferin from cut material (method 14) was only around $15 \%$ of that amount. The situations were similar between powdered 
and cut material when using boiling temperature extraction with methanol (methods 16 and 17 , respectively).

More or less the same concentrations of trans-resveratrol were obtained by maceration at the laboratory temperature after seven days in powdered (method 9) as in cut material (method 8), but differences between trans-e-viniferin and r2-viniferin yields were more than twofold. A statistical analysis is given in detail in Table 3 .

Table 3. Tukey's honest significance test for stilbenes (trans-resveratrol, trans-e-viniferin and $\mathrm{r} 2$-viniferin) in $\mu \mathrm{g} / \mathrm{g} \mathrm{d.w}$. for different particle sizes of grape cane. (Two-way ANOVA: ${ }^{* *} p<0.01, * p<0.05$, n.s. $=$ not significant. Values are means and standard deviations calculated from three measurements).

\begin{tabular}{ccccccc}
\hline Extraction & $\{14\}$ & $\{17\}$ & $\{8\}$ & $\{13\}$ & $\{16\}$ & $\{9\}$ \\
\hline Resveratrol & $\mathbf{3 1 5 2 . 3}$ & $\mathbf{2 5 1 1 . 1}$ & $\mathbf{4 1 8 9 . 0}$ & $\mathbf{5 3 2 4 . 1}$ & $\mathbf{4 8 6 8 . 5}$ & $\mathbf{4 1 0 9 . 0}$ \\
Viniferin & $\mathbf{8 6 9 . 8}$ & $\mathbf{7 2 7 . 3}$ & $\mathbf{5 6 3 . 1}$ & $\mathbf{2 0 7 4 . 1}$ & $\mathbf{1 7 7 6 . 7}$ & $\mathbf{1 3 8 8 . 8}$ \\
r2-viniferin & $\mathbf{7 8 . 1}$ & $\mathbf{6 9 . 1}$ & $\mathbf{1 0 8 . 7}$ & $\mathbf{5 0 7 . 5}$ & $\mathbf{4 2 2 . 7}$ & $\mathbf{2 8 6 . 2}$ \\
\hline
\end{tabular}

$\{14\}$

FBE $\mathrm{MeOH}$

\begin{tabular}{|c|c|c|c|c|c|}
\hline$\frac{(\mathrm{C})}{\{17\}}$ & n.s. & & & & \\
\hline Reflux $\mathrm{MeOH}$ & n.s. & & & & \\
\hline $1 \mathrm{~h}(\mathrm{C})$ & n.s. & & & & \\
\hline$\{8\}$ & $* *$ & $* *$ & & & \\
\hline Lab. T. MeOH & $* *$ & n.s. & & & \\
\hline $7 \mathrm{~d}(\mathrm{C})$ & n.s. & n.s. & & & \\
\hline$\{13\}$ & $* *$ & $* *$ & $* *$ & & \\
\hline FBE $\mathrm{MeOH}$ & $* *$ & $* *$ & $* *$ & & \\
\hline$(\mathrm{P})$ & $* *$ & $* *$ & $* *$ & & \\
\hline$\{16\}$ & $* *$ & $* *$ & n.s. & n.s. & \\
\hline Reflux $\mathrm{MeOH}$ & $* *$ & $* *$ & $* *$ & $* *$ & \\
\hline $1 \mathrm{~h}(\mathrm{P})$ & $* *$ & $* *$ & $* *$ & $*$ & \\
\hline$\{9\}$ & $* *$ & $* *$ & n.s. & $* *$ & * \\
\hline Lab. T. MeOH & $* *$ & $* *$ & $* *$ & $* *$ & $* *$ \\
\hline $7 \mathrm{~d}(\mathrm{P})$ & $* *$ & $* *$ & $* *$ & $* *$ & $* *$ \\
\hline
\end{tabular}

\subsubsection{Temperature}

Comparison of maceration at laboratory temperature (treatment 9) with extraction at a higher temperature $\left(50{ }^{\circ} \mathrm{C}\right.$, treatment 11$)$ or with other extractions at the boiling point of the solvents (methanol $64.7{ }^{\circ} \mathrm{C}$ and acetone $56.5{ }^{\circ} \mathrm{C}$ ) or with ASE while performing the extraction at $100{ }^{\circ} \mathrm{C}$ (treatment 25) shows that trans- $\varepsilon$-viniferin and $\mathrm{r} 2$-viniferin had smaller yields at laboratory temperature than at higher temperature. Although the yields were similarly lower for the extraction of trans-resveratrol at laboratory temperature using acetone (e.g., treatment 4 versus treatments 10, 12 and 15), when using methanol, the values for trans-resveratrol by maceration at the laboratory 
temperature (e.g. treatment 8) were nearly the same as at a higher temperature (e.g. treatments 13 and 16). A statistical analysis is given in detail in Table 4.

Table 4. Tukey's honest significance test for stilbenes (trans-resveratrol, trans- $\varepsilon$-viniferin and $\mathrm{r} 2$-viniferin) in $\mu \mathrm{g} / \mathrm{g}$ d.w. for different extraction temperatures. (Two-way ANOVA: ** $p<0.01, * p<0.05$, n.s. $=$ not significant. Values are means and standard deviations calculated from three measurements).

\begin{tabular}{|c|c|c|c|c|c|c|c|}
\hline Extraction & $\{11\}$ & $\{25\}$ & $\{9\}$ & $\{13\}$ & $\{16\}$ & $\{19\}$ & $\{24\}$ \\
\hline Resveratrol & 5521.6 & 6032.3 & 4189.0 & 5324.1 & 4868.5 & 5170.5 & 5505.7 \\
\hline Viniferin & 2260.3 & 2059.8 & 563.1 & 2074.1 & 1776.7 & 1822.8 & 1962.5 \\
\hline r2-viniferin & 496.9 & 414.0 & 108.7 & 507.5 & 422.7 & 282.9 & 418.4 \\
\hline \multirow{3}{*}{\multicolumn{8}{|c|}{$\begin{array}{c}\{11\} \\
50{ }^{\circ} \mathrm{C} \mathrm{MeOH} \\
(\mathrm{P})\end{array}$}} \\
\hline & & & & & & & \\
\hline & & & & & & & \\
\hline$\{25\}$ & n.s. & & & & & & \\
\hline ASE MeOH & n.s. & & & & & & \\
\hline$(\mathrm{P})$ & n.s. & & & & & & \\
\hline$\{9\}$ & $*$ & $* *$ & & & & & \\
\hline Lab. T. MeOH & $* *$ & $* *$ & & & & & \\
\hline $7 \mathrm{~d}(\mathrm{P})$ & $* *$ & $* *$ & & & & & \\
\hline$\{13\}$ & n.s. & n.s. & n.s. & & & & \\
\hline FBE MeOH & n.s. & n.s. & $* *$ & & & & \\
\hline$(\mathrm{P})$ & n.s. & n.s. & $* *$ & & & & \\
\hline$\{16\}$ & n.s. & n.s. & n.s. & n.s. & & & \\
\hline Reflux $\mathrm{MeOH}$ & n.s. & n.s. & $* *$ & n.s. & & & \\
\hline $1 \mathrm{~h}(\mathrm{P})$ & n.s. & n.s. & $* *$ & n.s. & & & \\
\hline$\{19\}$ & n.s. & n.s. & n.s. & n.s. & n.s. & & \\
\hline Soxhlet $\mathrm{MeOH}$ & n.s. & n.s. & $* *$ & n.s. & n.s. & & \\
\hline $1 \mathrm{st}(\mathrm{P})$ & $* *$ & n.s. & $* *$ & $* *$ & $*$ & & \\
\hline$\{24\}$ & n.s. & n.s. & $*$ & n.s. & n.s. & n.s. & \\
\hline MAE MeOH & n.s. & n.s. & $* *$ & n.s. & n.s. & n.s. & \\
\hline$(\mathrm{P})$ & n.s. & n.s. & $* *$ & n.s. & n.s. & $*$ & \\
\hline
\end{tabular}

\subsubsection{Extraction Time}

Maceration at laboratory temperature and extraction at boiling point temperatures (reflux) were performed for different times. In the case of maceration at laboratory temperature, the time range included $8 \mathrm{~h}$ as well as two, four and seven days. The concentrations of all compounds were increasing until day 4, at which the maximum concentration was reached, and the concentrations were slightly decreasing thereafter until day 7. This phenomenon was observed for both solvents, acetone and methanol. In the case of extraction at the boiling point temperature for 1 and $2 \mathrm{~h}$, the contents of trans-resveratrol and trans- $\varepsilon$-viniferin were slightly increased at the longer boiling time while the concentration of r2-viniferin somewhat decreased. A statistical analysis is given in Table 5 and in detail in Figure 3. 


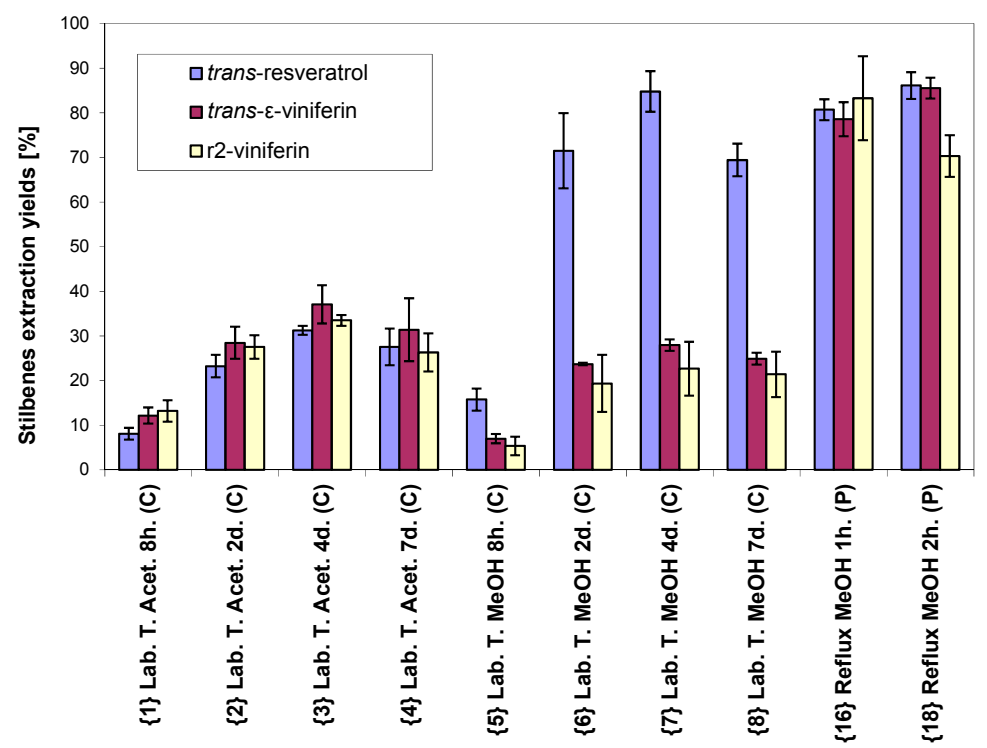

Figure 3. Comparison of stilbenes extraction yields in relation to extraction time (abbreviations are described in Table 1).

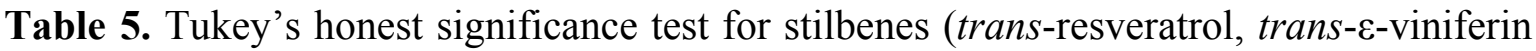
and $\mathrm{r} 2$-viniferin) in $\mu \mathrm{g} / \mathrm{g} \mathrm{d} . \mathrm{w}$. for different extraction times. (Two-way ANOVA: ** $p<0.01, * p<0.05$, n.s. $=$ not significant. Values are means and standard deviations calculated from three measurements)

\begin{tabular}{|c|c|c|c|c|c|c|c|c|}
\hline Extraction & $\{5\}$ & $\{1\}$ & $\{6\}$ & $\{2\}$ & $\{7\}$ & $\{3\}$ & $\{8\}$ & $\{4\}$ \\
\hline Resveratrol & 951.4 & 486.9 & 4314.1 & 1401.9 & 5115.4 & 1884.9 & 4189.0 & 1663.3 \\
\hline Viniferin & 157.9 & 275.2 & 536.0 & 643.7 & 632.1 & 838.2 & 563.1 & 709.5 \\
\hline r2-viniferin & 27.2 & 67.0 & 98.3 & 139.8 & 115.1 & 170.1 & 108.7 & 133.5 \\
\hline \multicolumn{9}{|l|}{$\begin{array}{c}\{5\} \\
\text { Lab. T. MeOH } \\
8 \mathrm{~h}(\mathrm{C})\end{array}$} \\
\hline$\{1\}$ & n.s. & & & & & & & \\
\hline $\begin{array}{c}\text { Lab. T. Acet. } \\
\quad 8 \text { h (C) }\end{array}$ & $\begin{array}{l}\text { n.s. } \\
\text { n.s. }\end{array}$ & & & & & & & \\
\hline$\{6\}$ & $* *$ & $* *$ & & & & & & \\
\hline Lab. T. MeOH & $* *$ & $*$ & & & & & & \\
\hline $2 \mathrm{~d}(\mathrm{C})$ & $*$ & n.s. & & & & & & \\
\hline$\{2\}$ & n.s. & $* *$ & $* *$ & & & & & \\
\hline Lab. T. Acet. & $* *$ & $* *$ & n.s. & & & & & \\
\hline $2 \mathrm{~d}(\mathrm{C})$ & $* *$ & $*$ & n.s. & & & & & \\
\hline$\{7\}$ & $* *$ & $* *$ & $*$ & $* *$ & & & & \\
\hline Lab. T. MeOH & $* *$ & $* *$ & n.s. & n.s. & & & & \\
\hline $4 \mathrm{~d}(\mathrm{C})$ & $* *$ & n.s. & n.s. & n.s. & & & & \\
\hline$\{3\}$ & $* *$ & $* *$ & $* *$ & n.s. & $* *$ & & & \\
\hline Lab. T. Acet. & $* *$ & $* *$ & $* *$ & n.s. & n.s. & & & \\
\hline $4 d(C)$ & $* *$ & $* *$ & $*$ & n.s. & n.s. & & & \\
\hline$\{8\}$ & $* *$ & $* *$ & n.s. & $* *$ & $* *$ & $* *$ & & \\
\hline Lab. T. MeOH 7 & $* *$ & $* *$ & n.s. & n.s. & n.s. & $* *$ & & \\
\hline $\mathrm{d}(\mathrm{C})$ & $* *$ & n.s. & n.s. & n.s. & n.s. & $*$ & & \\
\hline$\{4\}$ & $*$ & $* *$ & $* *$ & n.s. & $* *$ & n.s. & $* *$ & \\
\hline Lab. T. Acet. & $* *$ & $* *$ & n.s. & n.s. & n.s. & n.s. & n.s. & \\
\hline $7 \mathrm{~d}(\mathrm{C})$ & $* *$ & $*$ & n.s. & n.s. & n.s. & n.s. & n.s. & \\
\hline
\end{tabular}




\subsubsection{Multiple Extractions}

Multiple extractions were performed by Soxhlet extraction using methanol. The first extraction step (with 10 cycles) had much higher yields compared to the second, third, fourth and fifth steps. The yields of all three compounds by the second step were under $5 \%$, and the yields of the third and further steps were under 1\%. A statistical analysis is given in Table 6 and in detail in Figure 4.

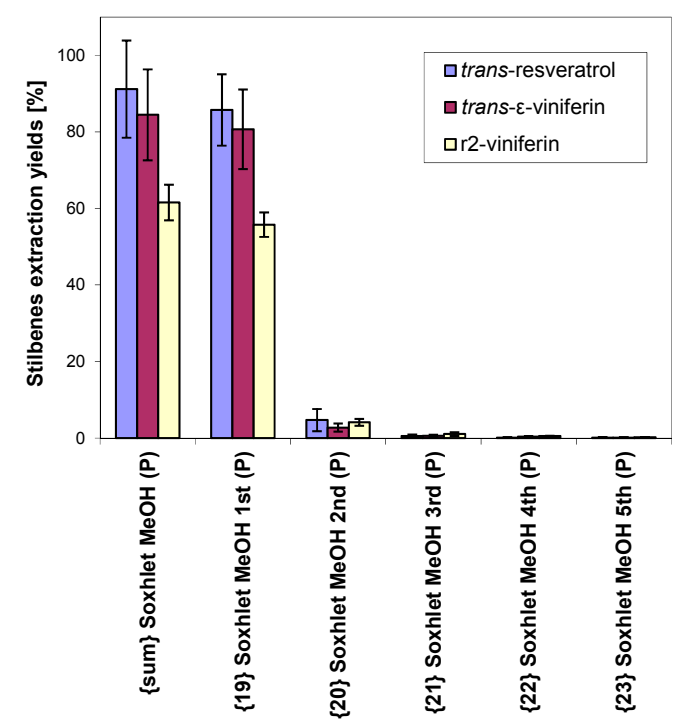

Figure 4. Comparison of stilbenes extraction yields in relation to number of extraction steps (abbreviation $\{\mathrm{sum}\}$ refers to quantity from all five steps together, other abbreviations are described in Table 1).

Table 6. Tukey's honest significance test for stilbenes (trans-resveratrol, trans- $\varepsilon$-viniferin and $\mathrm{r} 2$-viniferin) in $\mu \mathrm{g} / \mathrm{g} \mathrm{d} . \mathrm{w}$. for consecutive extraction steps. (Two-way ANOVA: ** $p<0.01, * p<0.05$, n.s. $=$ not significant. Values are means and standard deviations calculated from three measurements)

\begin{tabular}{|c|c|c|c|c|c|}
\hline Extraction & $\{19\}$ & $\{20\}$ & $\{21\}$ & $\{22\}$ & $\{23\}$ \\
\hline Resveratrol & 5170.5 & 282.2 & 29.9 & 8.2 & 7.7 \\
\hline Viniferin & 1822.8 & 61.5 & 13.3 & 8.3 & 2.8 \\
\hline r2-viniferin & 282.9 & 20.7 & 5.2 & 2.5 & 0.8 \\
\hline \multirow{3}{*}{\multicolumn{6}{|c|}{$\begin{array}{c}\{19\} \\
\text { Soxhlet } \mathrm{MeOH} \\
1 \mathrm{st}(\mathrm{P}) \\
\end{array}$}} \\
\hline & & & & & \\
\hline & & & & & \\
\hline$\{20\}$ & $* *$ & & & & \\
\hline Soxhlet $\mathrm{MeOH}$ & $* *$ & & & & \\
\hline 2nd $(\mathrm{P})$ & $* *$ & & & & \\
\hline$\{21\}$ & $* *$ & n.s. & & & \\
\hline Soxhlet $\mathrm{MeOH}$ & $* *$ & n.s. & & & \\
\hline 3rd (P) & $* *$ & n.s. & & & \\
\hline$\{22\}$ & $* *$ & n.s. & n.s. & & \\
\hline Soxhlet $\mathrm{MeOH}$ & $* *$ & n.s. & n.s. & & \\
\hline 4th $(\mathrm{P})$ & $* *$ & n.s. & n.s. & & \\
\hline$\{23\}$ & $* *$ & n.s. & n.s. & n.s. & \\
\hline Soxhlet $\mathrm{MeOH}$ & $* *$ & n.s. & n.s. & n.s. & \\
\hline 5th $(\mathrm{P})$ & $* *$ & n.s. & n.s. & n.s. & \\
\hline
\end{tabular}




\subsubsection{Extraction Efficiency}

Extraction efficiency was influenced by numerous factors-drying, grinding, temperature, extraction time, type of solvent and the extraction procedure (specified in Sections 2.3.1-2.3.4). ASE with methanol and powdered material—extraction method 25 was the most effective treatment (see Table 1).

Extraction method $11\left(50{ }^{\circ} \mathrm{C}\right.$, methanol, powdered material), extraction method 13 (FBE, boiling temperature, methanol, powdered material) and also extraction method 24 (MAE, boiling temperature, methanol, powdered material) were comparable with the extraction using ASE.

Extraction of cut material using the same instruments and procedure reached only about $50 \%$ as compared with powdered material.

Extraction method 1 (laboratory temperature, acetone, eight hours, cut material) achieved only about $10 \%$ efficiency compared to the ASE treatment.

\subsection{Quantity and Quality of Stilbenes}

\subsubsection{Determination of Stilbenes}

Only three main peaks of resveratrol and its derivatives having fluorescence were analyzed by HPLC-DAD/FLD at $220 \mathrm{~nm}$ and low resolution LC-DAD/MS (see Figure 5). Next to these method HRMS (High Resolution Mass Spectrometry) and LC-NMR for identification of both viniferins were used.

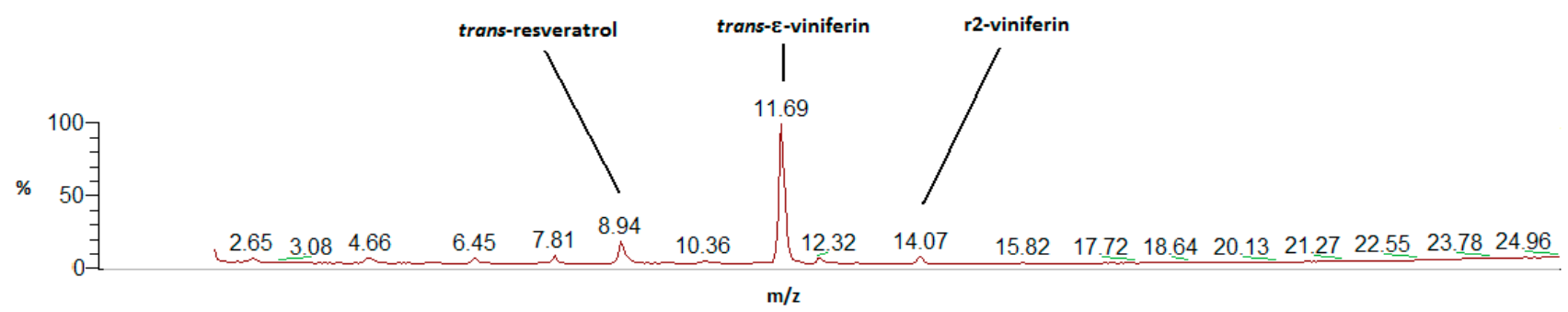

Figure 5. Chromatogram of all three stilbenes (extraction No. 11) in full-scan (positive mode at atmospheric pressure chemical ionization-APCI).

In the samples measured by LC-MS, there is a minor peak at the retention time of 12.32 min with $m / z=681$ and with small fluorescence in the HPLC-DAD/FLD system. This compound could be one of the resveratrol trimers (e.g. amurensin $\mathrm{B}$, amurensin $\mathrm{G}$ or gnetin $\mathrm{H}$ ).

\subsubsection{Identification of Viniferins}

In contrast to trans-resveratrol whose standard was available, trans- $\varepsilon$-viniferin $(\mathrm{MW}=454$, $\left.\mathrm{t}_{\mathrm{R}}=11.69 \mathrm{~min}\right)$ and $\mathrm{r} 2$-viniferin $\left(\mathrm{MW}=906, \mathrm{t}_{\mathrm{R}}=14.07 \mathrm{~min}\right)$ were identified by HRMS and LC-NMR. Monoisotopic masses of molecular ions and fragment ions for both viniferins from high-resolution mass spectrometry measurements are given in the Table 7. 
Table 7. Monoisotopic masses for viniferins in Daltons (full-scan and MS/MS mode)

\begin{tabular}{|c|c|c|c|c|c|}
\hline \multicolumn{3}{|c|}{ 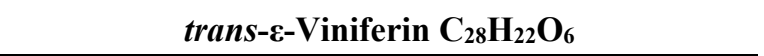 } & \multicolumn{3}{|c|}{ r2-Viniferin $\mathrm{C}_{56} \mathrm{H}_{42} \mathrm{O}_{12}$} \\
\hline Formula & Found $[m / z]$ & $\begin{array}{c}\text { Calculated } \\
{[m / z]}\end{array}$ & Formula & Found $[m / z]$ & Calculated $[m / z]$ \\
\hline $\mathrm{C}_{28} \mathrm{H}_{23} \mathrm{O}_{6}{ }^{+}[\mathrm{M}+\mathrm{H}]^{+}$ & 455.1482 & 455.1489 & $\mathrm{C}_{56} \mathrm{H}_{43} \mathrm{O}_{12}{ }^{+}[\mathrm{M}+\mathrm{H}]^{+}$ & 907.2745 & 907.2749 \\
\hline $\mathrm{C}_{28} \mathrm{H}_{21} \mathrm{O}_{5}^{+}\left[\mathrm{M}-\mathrm{H}_{2} \mathrm{O}\right)^{+}$ & 437.1373 & 437.1383 & {$[\mathrm{M}+\mathrm{Na}]^{+}$} & 929.2960 & 929.2968 \\
\hline $\mathrm{C}_{22} \mathrm{H}_{17} \mathrm{O}_{5}^{+}\left[\mathrm{M}-\mathrm{C}_{6} \mathrm{H}_{5} \mathrm{O}\right]^{+}$ & 361.0740 & 361.070 & {$[\mathrm{M}+\mathrm{K}]^{+}$} & 945.2305 & 945.2307 \\
\hline \multirow[t]{4}{*}{$\mathrm{C}_{13} \mathrm{H}_{11} \mathrm{O}_{3}^{+}$} & 215.0709 & 215.0702 & $\mathrm{C}_{35} \mathrm{H}_{27} \mathrm{O}_{7}^{+}$ & 559.1709 & 559.1751 \\
\hline & & & $\mathrm{C}_{28} \mathrm{H}_{21} \mathrm{O}_{6}^{+}$ & 453.1339 & 453.1332 \\
\hline & & & $\mathrm{C}_{22} \mathrm{H}_{17} \mathrm{O}_{5}^{+}$ & 361.1038 & 361.1070 \\
\hline & & & $\mathrm{C}_{13} \mathrm{H}_{11} \mathrm{O}_{3}{ }^{+}$ & 215.0690 & 215.0702 \\
\hline
\end{tabular}

The obtained UV spectrum of trans- $\varepsilon$-viniferin and $\mathrm{r} 2$-viniferin in the water-acetonitrile mixture acidified with $0.1 \%$ of $o$-phosphoric acid revealed the following maxima: trans- $\varepsilon$-viniferin $-\lambda$ max: 226 and $324 \mathrm{~nm}$; r2-viniferin - $\lambda_{\max }$ : 226 and $326 \mathrm{~nm}$. Ha et al. [13] have reported UV spectrum of trans- $\varepsilon$-viniferin in methanol ( $\lambda_{\max }: 226,285$ and $\left.322 \mathrm{~nm}\right)$ and for r2-viniferin in methanol $\left(\lambda_{\max }: 224\right.$, 285 and $327 \mathrm{~nm})$.

NMR identification: The structure elucidation of trans- $\varepsilon$-viniferin and $\mathrm{r} 2$-viniferin was based on ${ }^{1} \mathrm{H}$ and COSY 2D experiment and comparison of literature data $[14,15]$. The ${ }^{13} \mathrm{C}-\mathrm{NMR}$ experiment could not be performed (neither direct nor indirect) to further confirm compound structure due to low concentration. Therefore, the assignment of NMR signals was confronted with the predicted ${ }^{1} \mathrm{H}-\mathrm{NMR}$ spectrum using current version of NMR predictor software [16] and showed good agreement. Some signals were hidden under signal of water; these signals were located from COSY spectrum. The ultimate confirmation of the structure was provided by off-line HRMS performed on trapped chromatographic peaks.

trans- $\varepsilon$-Viniferin: ${ }^{1} \mathrm{H}-\mathrm{NMR}: \delta(\mathrm{ppm}) 7.18\left(\mathrm{~d}, 2 \mathrm{H}, \mathrm{H}-2, \mathrm{H}-6, J_{2,3}=J_{5,6}=8.5 \mathrm{~Hz}\right) 7.15(\mathrm{~d}, 2 \mathrm{H}, \mathrm{H}-2$ ', H-6', $\left.J_{2^{\prime}, 3^{\prime}}=J_{5,6^{\prime}}=8.6 \mathrm{~Hz}\right), 6.92\left(\mathrm{~d}, 1 \mathrm{H}, \mathrm{H}-8^{\prime}, J_{7^{\prime}, 8^{\prime}}=16.4 \mathrm{~Hz}\right), 6.82\left(\mathrm{~d}, 2 \mathrm{H}, \mathrm{H}-3, \mathrm{H}-5, J_{2,3}=J_{5,6}=8.5 \mathrm{~Hz}\right)$, $6.74\left(\mathrm{~d}, 2 \mathrm{H}, \mathrm{H}-3^{\prime}, \mathrm{H}-5^{\prime}, J_{2^{\prime}, 3^{\prime}}=J_{6^{\prime}, 5^{\prime}}=8.6 \mathrm{~Hz}\right), 6.68\left(\mathrm{~d}, 1 \mathrm{H}, \mathrm{H}-14^{\prime}, J_{12^{\prime}, 14^{\prime}}=1.8 \mathrm{~Hz}\right), 6.62\left(\mathrm{~d}, 1 \mathrm{H}, \mathrm{H}-7^{\prime}\right.$, $\left.J_{7^{\prime}, 8^{\prime}}=16.4 \mathrm{~Hz}\right), 6.33\left(\mathrm{~d}, 1 \mathrm{H}, \mathrm{H}-12^{\prime}, J_{12^{\prime}, 14^{\prime}}=1.8 \mathrm{~Hz}\right), 6.18\left(\mathrm{~d}, 2 \mathrm{H}, \mathrm{H}-10, \mathrm{H}-14, J_{10,12}=J_{12,14}=1.9 \mathrm{~Hz}\right)$, $6.16\left(\mathrm{t}, 1 \mathrm{H}, \mathrm{H}-12, J_{10,12}=J_{12,14}=1.9 \mathrm{~Hz}\right), 5.47\left(\mathrm{~d}, 1 \mathrm{H}, \mathrm{H}-7, J_{7,8}=6.0 \mathrm{~Hz}\right), 4.47(\mathrm{~d}, 1 \mathrm{H}, \mathrm{H}-8$, $\left.J_{7,8}=6.0 \mathrm{~Hz}\right)$.

r2-Viniferin: ${ }^{1} \mathrm{H}-\mathrm{NMR}: \delta$ (ppm) 7.20 (d, 2H, H-2, H-6, $\left.J_{2,3}=J_{5,6}=8.6 \mathrm{~Hz}\right) 7.18$ (d, 2H, H-2"', H-6"', $\left.J_{2^{\prime \prime \prime}, 3^{\prime \prime \prime}}=J_{5^{\prime \prime}, 6^{\prime \prime \prime}}=8.6 \mathrm{~Hz}\right), 7.10\left(\mathrm{~d}, 1 \mathrm{H}, \mathrm{H}-6{ }^{\prime}, J_{5^{\prime}, 6^{\prime}}=8.5 \mathrm{~Hz}\right), 6.83\left(\mathrm{~d}, 2 \mathrm{H}, \mathrm{H}-3, \mathrm{H}-5, J_{2,3}=J_{5,6}=8.6 \mathrm{~Hz}\right)$, 6.81 (d, 2H, H-3"', H-5"', $\left.J_{22^{\prime \prime}, 3^{\prime \prime}}=J_{66^{\prime \prime}, 5^{\prime \prime}}=8.6 \mathrm{~Hz}\right), 6.79$ (d, 1H, H-8', $\left.J_{7^{\prime}, 8^{\prime}}=16.2 \mathrm{~Hz}\right), 6.78$ (s, 1H, H-2'), 6.77 (d, 1H, H-5', $\left.J_{5^{\prime}, 6^{\prime}}=8.5 \mathrm{~Hz}\right), 6.64$ (d, 2H, H-2", H-6", $\left.J_{2^{\prime \prime}, 3^{\prime \prime}}=J_{5^{\prime \prime}, 6^{\prime \prime}}=8.6 \mathrm{~Hz}\right), 6.61$ (d, 1H, H-14', $\left.J_{12^{\prime}, 14^{\prime}}=1.9 \mathrm{~Hz}\right), 6.57$ (d, 2H, H-3", H-5", $\left.J_{2^{\prime \prime}, 3^{\prime \prime}}=J_{5^{\prime \prime}, 6^{\prime \prime}}=8.6 \mathrm{~Hz}\right), 6.55$ (d, 1H, H-7', $\left.J_{7^{\prime}, 8^{\prime}}=16.2 \mathrm{~Hz}\right), 6.34$ $\left(\mathrm{d}, 1 \mathrm{H}, \mathrm{H}-12^{\prime}, J_{12^{\prime}, 14^{\prime}}=1.9 \mathrm{~Hz}\right), 6.31\left(\mathrm{~d}, 1 \mathrm{H}, \mathrm{H}-14^{\prime \prime \prime}, J_{122^{\prime \prime}, 14^{\prime \prime}}=2.0 \mathrm{~Hz}\right), 6.12$ (d, 1H, H-14",

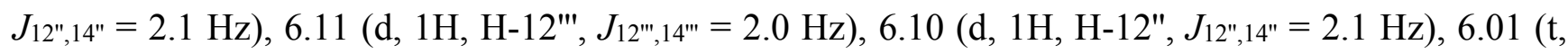
$\left.1 \mathrm{H}, \mathrm{H}-12, J_{10,12}=J_{12,14}=1.9 \mathrm{~Hz}\right), 5.95$ (d, 2H, H-10, H-14, $\left.J_{10,12}=J_{12,14}=1.9 \mathrm{~Hz}\right), 5.50\left(\mathrm{~d}, 1 \mathrm{H}, \mathrm{H}-7\right.$ '"', $^{\prime}$ $\left.J_{7^{\prime \prime}, 8^{\prime \prime \prime}}=5.5 \mathrm{~Hz}\right), 5.45\left(\mathrm{~d}, 1 \mathrm{H}, \mathrm{H}-7^{\prime \prime}, J_{7^{\prime \prime}, 8^{\prime \prime}}=5.5 \mathrm{~Hz}\right), 5.38\left(\mathrm{~d}, 1 \mathrm{H}, \mathrm{H}-7, J_{7,8}=5.5 \mathrm{~Hz}\right), 4.46$ (d, 1H, H-8", $\left.J_{7^{\prime \prime}, 8^{\prime \prime}}=5.5 \mathrm{~Hz}\right), 4.46\left(\mathrm{~d}, 1 \mathrm{H}, \mathrm{H}-8, J_{7,8}=5.5 \mathrm{~Hz}\right), 4.25$ (d, $\left.1 \mathrm{H}, \mathrm{H}-8^{\prime \prime \prime}, J_{7^{\prime \prime}, 8^{\prime \prime \prime}}=5.5 \mathrm{~Hz}\right)$. 


\section{Experimental Section}

\subsection{Standards and Solvents}

Standard of trans-resveratrol was purchased from (Sigma-Aldrich, Prague, Czech Republic) and measured as solution in pure methanol. Solvents were obtained as follows: methanol, acetone and acetonitrile (Merck, Prague, Czech Republic, LiChrosolv, gradient grade for LC), acetonitrile (Fisher Scientific, Pardubice, Czech Republic, Optima LC/MS), ortho-phosporic acid (Fluka, Prague, Czech Republic, p.a.) and formic acid (Sigma-Aldrich, Prague, Czech Republic, gradient grade).

\subsection{Samples Analysis}

\subsubsection{High-Performance Liquid Chromatography}

The samples were analyzed using an HP 1050 (Ti-series) HPLC instrument (Hewlett Packard, Palo Alto, CA, USA) on a $3 \mu \mathrm{m}, 150 \mathrm{~mm} \times 2 \mathrm{~mm}$, Luna C18(2) column (Phenomenex, Torrance, CA, USA) with water-acetonitrile-o-phosphoric acid mobile phase. Mobile phase A used 5\% of acetonitrile $+0.1 \%$ of $o$-phosphoric acid; mobile phase B used $80 \%$ of acetonitrile $+0.1 \%$ of $o$-phosphoric acid (in vol. $\%$ ). The gradient was increased from $20 \%$ of B to $80 \%$ of B during 20 min and from $80 \%$ of B to $100 \%$ of B during $5 \mathrm{~min}$. Flow rate was $0.250 \mathrm{~mL} / \mathrm{min}$ and column temperature $25{ }^{\circ} \mathrm{C}$. Injection volume was $5 \mu \mathrm{L}$. Also used were an G1315B diode array detector (DAD, Agilent, Prague, Czech Republic) with detection wavelengths at 220 and $315 \mathrm{~nm}$ and scanning range 190-600 nm, as well as an G1321A fluorescence detector (FLD, Agilent, Prague, Czech Republic) with excitation wavelength $315 \mathrm{~nm}$, emission wavelength $395 \mathrm{~nm}$, and scanning of emission in the range of 300-600 nm. Finally, the method was validated in terms of linearity, limits of detection, and repeatability $[17,18]$.

\subsubsection{Liquid Chromatography-Mass Spectrometry (LC-MS)}

Low-resolution LC-MS measurement was performed using an LCQ Accela Fleet (Thermo Fisher Scientific, San Jose, CA, USA) equipped with electro-spray (ESI), atmospheric pressure chemical (APCI), and atmospheric pressure photo (APPI) ionization sources and a photodiode array detector. A $3 \mu \mathrm{m}, 150 \mathrm{~mm} \times 2 \mathrm{~mm}$, Luna C18(2) column (Phenomenex, Torrance, CA, USA) was used with water-acetonitrile-formic acid mobile phase. Mobile phase A used 5\% of acetonitrile $+0.1 \%$ of formic acid; mobile phase $\mathrm{B}$ used $80 \%$ of acetonitrile $+0.1 \%$ of formic acid (in vol.\%). The gradient was increased from $20 \%$ of B to $80 \%$ of B during $20 \mathrm{~min}$ and from $80 \%$ of B to $100 \%$ of B during $5 \mathrm{~min}$. Injection volume was $10 \mu \mathrm{L}$ and flow rate $0.400 \mathrm{~mL} / \mathrm{min}$. APCI capillary temperature was $275{ }^{\circ} \mathrm{C}$, APCI vaporizer temperature $400{ }^{\circ} \mathrm{C}$, sheath gas flow $58 \mathrm{~L} / \mathrm{min}$, auxiliary gas flow $10 \mathrm{~L} / \mathrm{min}$, source voltage $6 \mathrm{kV}$, source current $5 \mu \mathrm{A}$, and capillary voltage $10 \mathrm{~V}[17,18]$.

\subsubsection{Liquid Chromatography- Nuclear Magnetic Resonance (LC-NMR)}

A commercial HPLC system (Dionex UltiMate 3000, Thermo Fisher Scientific, San Jose, CA, USA) with $250 \mathrm{~mm} \times 4.6 \mathrm{~mm}$ HPLC column (Luna C18(2), Phenomenex, $5 \mu \mathrm{m}$ particles, $100 \AA$ pore size) was employed. Fifty microliters of the concentrated methanol solution was injected into HPLC. 
The separation was done by gradient method described in the HPLC section (see Section 3.2.2.; system acetonitrile- $\mathrm{D}_{2} \mathrm{O}$ ) and was monitored at $220 \mathrm{~nm}$ and by on-flow ${ }^{1} \mathrm{H}-\mathrm{NMR}$ detection. ${ }^{1} \mathrm{H}-\mathrm{NMR}$ observations were conducted on Varian INOVA $500 \mathrm{MHz}$ spectrometer (Varian Inc., Palo Alto, CA, USA) equipped with HCN triple resonance (60 $\mu \mathrm{L}$ active volume) microflow probe. Standard NMR software VnmrJ 4.2 was used. All the separations and NMR detection were conducted at ambient temperature $\left(22{ }^{\circ} \mathrm{C}\right)$. The ${ }^{1} \mathrm{H}-\mathrm{NMR}$ data were collected in on-flow mode employing WET multiple frequency solvent suppression [19]. The signal of acetonitrile solvent ( $\delta=2.00 \mathrm{ppm})$ was suppressed using one scout scan prior to whole data collection. The data acquisition during $1 \mathrm{~s}$ acquisition time covering the spectral width of $6 \mathrm{kHz}$ followed after $90^{\circ} \mathrm{RF}$ pulse $(3.4 \mu \mathrm{s})$, four transients were accumulated in each spectrum. No relaxation delay was employed.

The detailed analysis of chromatographic peaks was performed in the stop-flow mode. ${ }^{1} \mathrm{H}-\mathrm{NMR}$ spectra were accumulated over 128 scans (acquisition time $2 \mathrm{~s}$, relaxation delay $1 \mathrm{~s}$ ). COSY experiment took $2 \mathrm{~h}$ for trans- $\varepsilon$-viniferin and 12 hours for $\mathrm{r} 2$-viniferin. The ${ }^{1} \mathrm{H}-\mathrm{NMR}$ spectra with assigned structures of trans- $\varepsilon$-viniferin and r2-viniferin are depicted in the Figures 6 and 7.

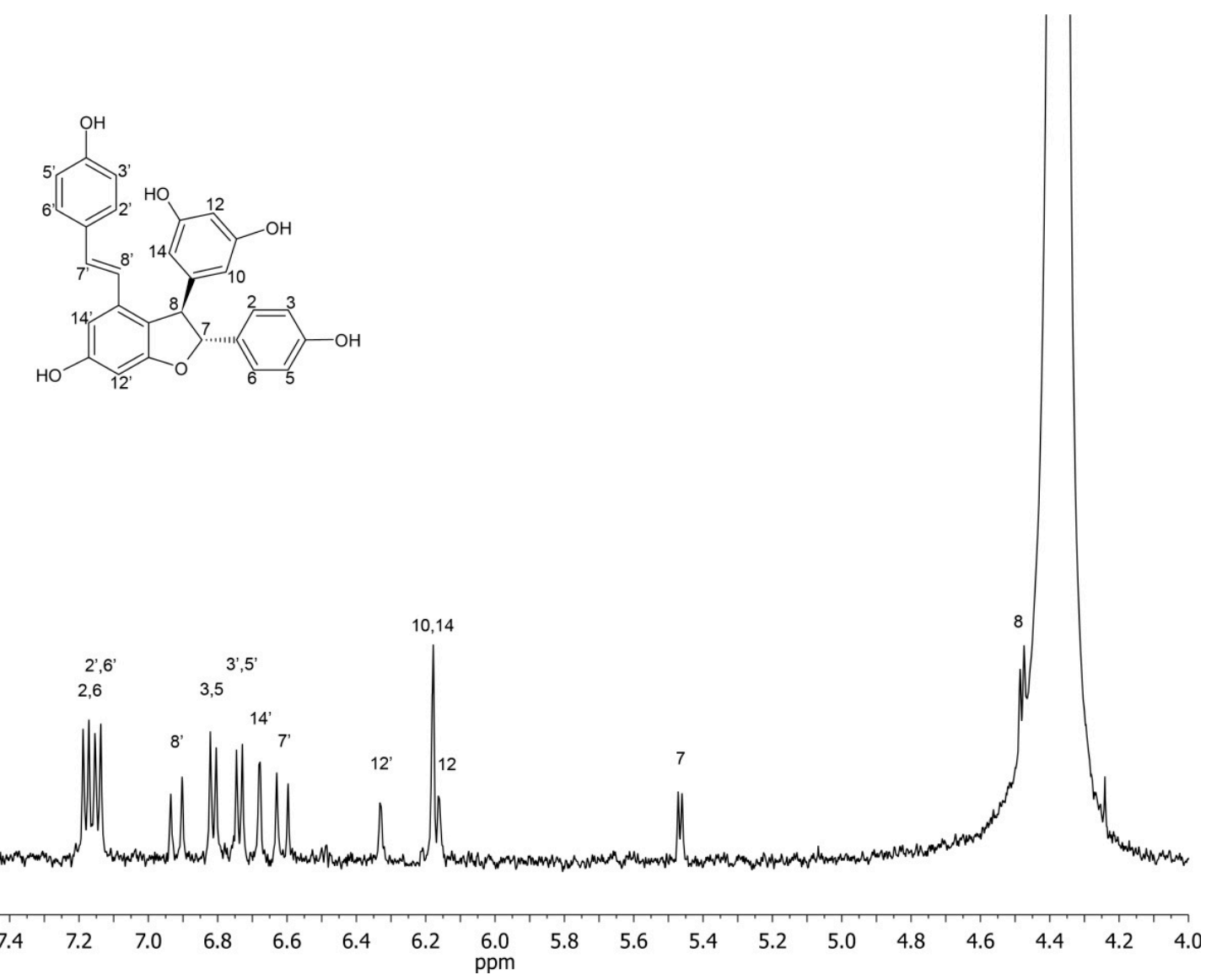

Figure 6. ${ }^{1} \mathrm{H}-\mathrm{NMR}$ spectrum and signal assignment of trans- $\varepsilon$-viniferin. 

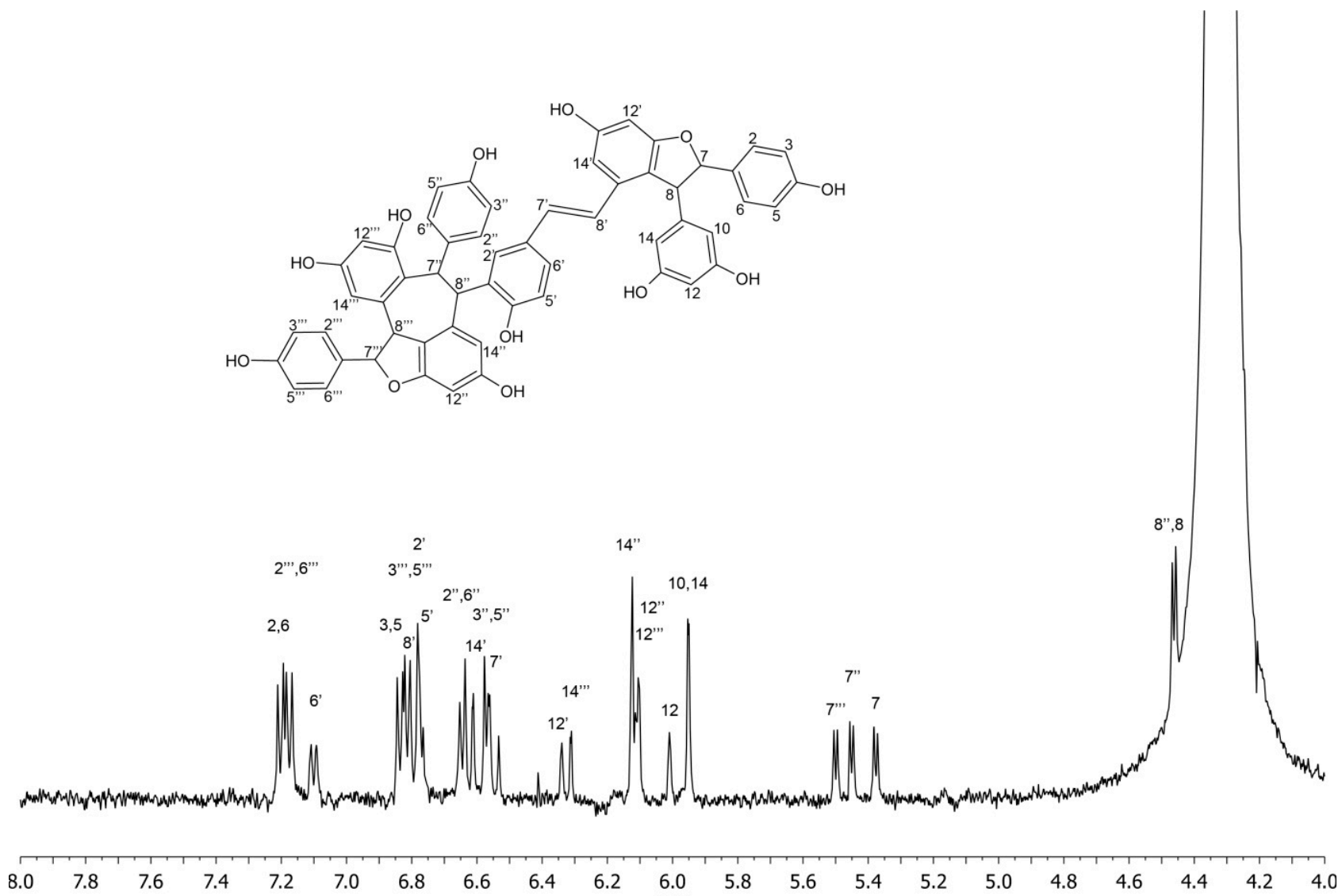

Figure 7. ${ }^{1} \mathrm{H}-\mathrm{NMR}$ spectrum and signal assignment of r2-viniferin. Signal of H-8'" is hidden under water signal at $4.25 \mathrm{ppm}$.

\subsubsection{High Resolution Mass Spectrometry (HRMS)}

The HRMS spectra of given samples were measured at Bruker MicrOTOF-QIII apparatus. Due to the structure of targeted compounds, the ESI source in the positive mode was used and the parameters of the measurement were adjusted as follows: the capillary voltage was $4200 \mathrm{~V}$, the end plate offset was $-500 \mathrm{~V}$. The collision cell RF was $350 \mathrm{Vpp}$. Nitrogen was used as the nebulizer gas (at the pressure of 1.6 bar), just as the drying gas (heated to $180{ }^{\circ} \mathrm{C}$, with the flow of $8 \mathrm{~L} / \mathrm{min}$ ). The scans of MS spectra were carried out in the mass range of $m / z$ 80-1550. For the HRMS measurement, the calibration on $\mathrm{Na}$-formate clusters was used.

The samples were delivered by the direct infusion using the syringe pump (Kd-Scientific, KDS-100-CE, $0.5 \mathrm{~mL}$ Hamilton syringe, flow $180 \mathrm{~mL} / \mathrm{min}$ ) coupled to the MicrOTOF-QIII mass spectrometer.

For the MS/MS measurements, the precursor ions were isolated by the collision cell energy $2 \mathrm{eV}$ in the window of 2 units $(\mathrm{m} / \mathrm{z})$ and the product ions were obtained at the collision cell energy rising from 25 to $35 \mathrm{eV}$, depending on the compound examined.

\subsection{Samples}

Samples were from Vitis vinifera L. cv. Cabernet Moravia (crossing: Cabernet Franc $\mathrm{x}$ Zweigeltrebe) grown in wine region Moravia, sub-region Slovacko, village Kostice (GPS: $48^{\circ} 44^{\prime} 50^{\prime \prime} \mathrm{N}$ 
$16^{\circ} 58^{\prime} 26^{\prime \prime} \mathrm{E}$ ). Grape canes were dried at room temperature for 2.5 months after pruning (March 2013) in darkness and lyophilization was used for final drying. Dried grape canes were then cut to length around $1.0 \mathrm{~cm}$ and one part of the canes was powdered in a knife grinder to an approximate size of $1 \mathrm{~mm}$. In this way, we obtained two types of plant samples with different particle sizes.

\subsection{Extraction Method}

We compared the following seven types of extraction methods in our experiments, for both powdered ( $>1 \mathrm{~mm})$ and cut $(\sim 1 \mathrm{~cm})$ material: (1) at laboratory temperature, (2) at $50{ }^{\circ} \mathrm{C}$, (3) at boiling point with a reflux condenser, (4) by fluidized-bed extraction, (5) by Soxhlet extraction, (6) by microwave-assisted extraction (MAE), and (7) by accelerated solvent extraction (ASE).

\subsubsection{Extraction at Laboratory Temperature}

We added $20 \mathrm{~mL}$ of solvent (acetone or methanol) to $2 \mathrm{~g}$ of dry grape canes in a flask for use in measuring stilbenes content after four consecutive extraction times. We later took four times $200 \mu \mathrm{L}$ samples of the material prepared using cut dry canes (not that from using powered canes) after each of 8 h, 2 days and 4 days of extraction. The samples were immediately stored in a refrigerator until analysis. Extractions were stopped on day 7 for both solvents for cut material. Powdered material was done similarly, but measured only one time on day 7 (without precious times: 8 h, 2 and 4 days). Solid portions were then separated by filtration, washed two times with $1 \mathrm{~mL}$ of solvent and then solvents were added to bring the final volume back to $20 \mathrm{~mL}$. Extractions were done at temperature was $22 \pm 1{ }^{\circ} \mathrm{C}$.

\subsubsection{Extraction at $50^{\circ} \mathrm{C}$}

We used $0.25 \mathrm{~g}$ of powdered grape canes and $3 \mathrm{~mL}$ of solvent for extraction at $50{ }^{\circ} \mathrm{C}$ during $165 \mathrm{~min}$. Smaller amounts of sample and solvent were used than in the laboratory temperature extraction but with roughly the same extraction ratio. After extraction, the mixture was centrifuged at $3500 \mathrm{rpm}$ for $10 \mathrm{~min}$ at $20{ }^{\circ} \mathrm{C}$. The supernatant was transferred into a calibrated tube, $1 \mathrm{~mL}$ of fresh solvent was added to the solid residue, mixed and this new mixture was centrifuged again. This rinse procedure was then repeated. As a result, the sample was extracted only once and the residue was washed two times with solvent. All supernatants were combined and the final volume of supernatant mixture was adjusted to $5 \mathrm{~mL}$.

\subsubsection{Extraction with Solvent Heated to Reflux}

The quantity, $1.50 \mathrm{~g}$ of grape canes, was transferred into a flask equipped with a reflux condenser and then $50 \mathrm{~mL}$ of methanol or acetone was added. The mixture was then heated to reflux at $70{ }^{\circ} \mathrm{C}$ in the water bath for 1 or $2 \mathrm{~h}$. The solution was filtered and then $1 \mathrm{~mL}$ of solvent was added into the flask and filtration was performed again through the same filter. One milliliter of solvent was used for washing (and then $2 \mathrm{~mL}$ again). Final volumes were adjusted to $50 \mathrm{~mL}$. In this way, we obtained solutions in acetone and methanol from powdered source material and a solution only in methanol from the cut source material. 
One milliliter from each of the acetone samples (from powdered material) was transferred into a calibrated tube. The acetone solution was dried by a flow of nitrogen gas at $45^{\circ} \mathrm{C}$, after which $1 \mathrm{~mL}$ of methanol was added to the dry residue for the analysis.

\subsubsection{Fluidized-Bed Extraction}

On each frit of an IKA fluidized-bed extractor's upper part (four vessels in total) we placed $1.5 \mathrm{~g}$ of grape cane material and $100 \mathrm{~mL}$ of solvent was placed into the bottom flask. The apparatus was heated to $140{ }^{\circ} \mathrm{C}$ for $20 \mathrm{~min}$, during which solvent vapors passed through the frit and became liquid on the condenser. Solvent from the condenser dripped onto the sample of grape canes. During extraction, new solvent vapor passed through the sample and in this manner increased its agitation. The cooling process was started after $20 \mathrm{~min}$ until the temperature had been reduced to $30{ }^{\circ} \mathrm{C}$. During this period, only the supernatant from the dispersed sample returned to the flask due to the lower pressure resulting from cooling of the solvent. Extraction in the fluidized-bed went through 5 cycles and final volumes were adjusted to $100 \mathrm{~mL}$. In this manner, we obtained methanol samples from powdered and from cut source material and acetone samples only from powdered source material.

\subsubsection{Soxhlet Extraction}

We placed $1.5 \mathrm{~g}$ of grape canes into the thimble in the extractor and $100 \mathrm{~mL}$ of methanol in the flask (acetone was not used), volume in Soxhlet apparatus was $50 \mathrm{~mL}$. We performed 10 cycles for each extraction step. One cycle means the time when the volume of supernatant in the thimble goes back into the flask. Completing these 10 cycles took a total time of around 2-3 h. We repeated this step with 10 cycles five times, then removed the supernatant for analysis and added $100 \mathrm{~mL}$ of new methanol for the new step. We performed five extraction steps for estimation of the total yield of selected stilbenes. For the sake of good detection and quantification, final volumes from this 5-step extraction were adjusted to $100 \mathrm{~mL}$ for the first two steps and to $3 \mathrm{~mL}$ for the last three steps.

\subsubsection{Microwave-Assisted Extraction (MAE)}

Using a 150 W MARS 6 Microwave Reaction System (CEM, Matthews, NC, USA), $1.5 \mathrm{~g}$ of powdered grape canes and $50 \mathrm{~mL}$ of methanol were placed into the cartridges and extracted. Ramp time was $20 \mathrm{~min}$ and hold time was $10 \mathrm{~min}$. After extraction, final volumes were adjusted back to $50 \mathrm{~mL}$.

\subsubsection{Accelerated Solvent Extraction (ASE)}

Extractions at high pressure $10-10.5 \mathrm{MPa}\left(1450-1520 \mathrm{psi}\right.$, respectively) and temperature of $100{ }^{\circ} \mathrm{C}$ were performed with $0.316 \mathrm{~g}, 0.386 \mathrm{~g}$ and $0.383 \mathrm{~g}$ of grape cane (powdered source material) in the ASE 350 Accelerated Solvent Extractor (Thermo Scientific, Dionex). Three 5 min cycles were used for methanol and the final volumes were $6.7 \mathrm{~mL}, 6.8 \mathrm{~mL}$ and $6.6 \mathrm{~mL}$ for analyses.

All types of measurements were performed with three parallel samples for statistical evaluation. All samples after extraction were immediately placed into a refrigerator. 


\subsection{Statistical Analysis}

All extracts of stilbenes were measured three times. Averages, standard deviations and $p<0.01$ or $p<0.05$ (significant $v s$. non-significant differences) were calculated and detailed in Tables $1-6$ and Figures 1-4. The data given in these tables were analyzed by ANOVA, applying the Tukey multiple range test for making comparisons with Statistica $\mathrm{Cz} 12$ and MS Excel 2010 software.

\section{Conclusions}

This work compared various extraction methods for obtaining viniferins and trans-resveratrol from grape cane of Vitis vinifera L. cv. Cabernet Moravia. The results show that the best extraction method for trans-resveratrol is accelerated solvent extraction using methanol and providing $6030 \pm 680 \mu \mathrm{g} / \mathrm{g} \mathrm{d}$.w. of trans-resveratrol it could be caused not only by temperature effect but also by pressure effect. Higher temperature extraction is better for trans- $\varepsilon$-viniferin with the yield of $2260 \pm 90 \mu \mathrm{g} / \mathrm{g} \mathrm{d}$.w. of that compound. For the last eluting stilbene, r2-viniferin, the maximum amount of $510 \pm 40 \mu \mathrm{g} / \mathrm{g} \mathrm{d}$. w. was obtained by fluidized-bed extraction. As expected, higher yields were obtained from the extractions with powdered source material than with cut material. There was a large difference in yields between trans-resveratrol $(>80 \%)$ and trans- $\varepsilon$-viniferin $(<30 \%)$ at laboratory temperature in the case of cut grape cane. This difference could be used for the separation and concentration of resveratrol. Next to the size of material, regarding extraction yield, multiple extraction has also afforded higher yield compared with single step extraction; similarly extraction at higher temperature is better than at laboratory temperature; in case of time, some maxima of yields were observed (four days at laboratory temperature for both solvents), but generally methanol was the better extraction solvent than acetone. The structure of obtained trans-e-viniferin and r2-viniferin was confirmed by NMR and high resolution mass spectrometry measurement.

\section{Acknowledgments}

Financial support for this research was provided by project Postdoc contracts at MENDELU, Technical and Economical Research Project No. CZ.1.07/2.3.00/30.0031 and by project No. LD14038 of the Ministry of Education, Youth and Sports of the Czech Republic.

\section{Author Contributions}

IS, NV, JT and JB designed research; IS and NV performed research and analyzed the data; identification of stilbenes were done by $\breve{S} \mathrm{H}$, PC and JS; IS, JT, JB, NV wrote the paper. All authors read and approved the final manuscript.

\section{Conflicts of Interest}

The authors declare no conflict of interest. 


\section{References}

1. Langcake, P.; Pryce, R.J. The production of resveratrol by Vitis vinifera and other members of the Vitaceae as a response to infection or injury. Physiol. Plant Pathol. 1976, 9, 77-86.

2. Sotheeswaran, S.; Pasupathy, V. Distribution of resveratrol oligomers in plants. Phytochemistry 1993, 32, 1083-1092.

3. Renaud, S.; de Lorgeril, M. Wine, alcohol, platelets, and the French paradox for coronary heart disease. Lancet 1992, 339, 1523-1526.

4. Catalgol, B.; Batirel, S.; Taga, Y.; Ozer, N.K. Resveratrol: French paradox revisited. Front. Pharmacol. 2012, 3, doi:10.3389/fphar.2012.00141.

5. Aaviksaar, A.; Haga, M.; Pussa, T.; Roasto, M.; Tsoupras, G. Purification of resveratrol from vine stems. Proc. Est. Acad. Sci.-Chem. 2003, 52, 155-164.

6. Pawlus, A.D.; Sahli, R.; Bisson, J.; Rivière, C.; Delaunay, J.C.; Richard, T.; Gomès, E.; Bordenave, L.; Waffo-Téguo, P.; Mérillon, J.M. Stilbenoid profiles of canes from Vitis and Muscadinia species. J. Agric. Food Chem. 2013, 61, 501-511.

7. Park, E.J.; Park, H.J.; Chung, H.J.; Shin, Y.; Min, H.Y.; Hong, J.Y.; Kang, Y.J.; Ahn, Y.H.; Pyee, J.H.; Lee, S.K. Antimetastatic activity of pinosylvin, a natural stilbenoid, is associated with the suppression of matrix metalloproteinases. J. Nutr. Biochem. 2012, 23, 946-952.

8. McCormack, D.; McFadden, D. A review of pterostilbene antioxidant activity and disease modification. Oxid. Med. Cell. Longev. 2013, 2013, 575482. doi:10.1155/2013/575482.

9. Zhang, L.; Cui, L.; Zhou, G.; Jing, H.; Guo, Y.; Sun, W. Pterostilbene, a natural small-molecular compound, promotes cytoprotective macroautophagy in vascular endothelial cells. J. Nutr. Biochem. 2013, 24, 903-911.

10. Balík, J.; Kyseláková, M.; Vrchotová, N.; Tř́ska, J.; Kumšta, M.; Veverka, J.; Híc, P.; Totušek, J.; Lefnerová, D. Relations between polyphenols content and antioxidant activity in vine grapes and leaves. Czech J. Food Sci. 2008, 26, S25-S32.

11. Rayne, S.; Karacabey, E.; Mazza, G. Grape cane waste as a source of trans-resveratrol and trans-viniferin: High-value phytochemicals with medicinal and anti-phytopathogenic applications. Ind. Crops Prod. 2008, 27, 335-340.

12. Vergara, C.; von Baer, D.; Mardones, C.; Wilkens, A.; Wernekinck, K.; Damm, A.; Macke, S.; Gorena, T.; Winterhalter, P. Stilbene levels in grape cane of different cultivars in southern Chile: Determination by HPLC-DAD-MS/MS method. J. Agric. Food Chem. 2012, 60, 929-933.

13. Ha, D.T.; Chen, Q.C.; Hung, T.M.; Youn, U.J.; Ngoc, T.M.; Thuong, P.T.; Kim, H.J.; Seong, Y.H.; Min, B.S.; Bae, K. Stilbenes and oligostilbenes from leaf and stem of Vitis amurensis and their cytotoxic activity. Arch. Pharm. Res. 2009, 32, 177-183.

14. Korhammer, S.; Reniero, F.; Mattivi, F. An oligostilbene from Vitis roots. Phytochemistry 1995, $38,1501-1504$.

15. Saputra, M.A.; Sirat, M.H.; Aminah, N.S. Stilbenoids from Vitis labrusca "Isabella" stems. Chem. Natur. Comp. 2013, 49, 924-926.

16. Mestrelab Research S.L., Spain, NMR program MestReNova, Version 8.1.4-12489. Available online: http://www.mestrelab.com (accessed on 2 April 2015). 
17. Tř́́ska, J.; Vrchotová, N.; Olejníčková, J.; Jílek, R.; Sotolář, R. Separation and identification of highly fluorescent compounds derived from trans-resveratrol in the leaves of Vitis vinifera infected by Plasmopara viticola. Molecules 2012, 17, 2773-2783.

18. Tř́iska, J.; Vrchotová, N.; Sýkora, J.; Moos, M. Separation and identification of 1,2,4-trihydroxynaphthalene-1-O-glucoside in Impatiens glandulifera Royle. Molecules 2013, 18 , 8429-8439.

19. Smallcombe, S.H.; Patt, S.L.; and Keifer, P.A. WET solvent suppression and its applications to LC NMR and high-resolution NMR spectroscopy. J. Magn. Reson. A 1995, 117, 295-303.

Sample Availability: Samples of the extracts are available from the authors.

(C) 2015 by the authors; licensee MDPI, Basel, Switzerland. This article is an open access article distributed under the terms and conditions of the Creative Commons Attribution license (http://creativecommons.org/licenses/by/4.0/). 